\title{
Ocupaciones de la Cueva de Ardales y Sima de las Palomas de Teba por sociedades neandertales
}

\author{
Occupations of Ardales Caves and Sima de las Palomas for by \\ Neardenthals societies
}

\author{
José Ramos-Muñoz \\ Universidad de Cádiz \\ Jose.ramos@uca.es \\ ORCID: https://orcid.org/0000-0002-6042-2446

\section{Gerd-C. Weniger} \\ Neanderthal Museum (Mettmann, Alemania) \\ weginer@neanderthal.de \\ ORCID: https://orcid.org/0000-0002-5270-657X \\ Pedro Cantalejo \\ Ayuntamiento de Ardales-Cueva de Ardales \\ pedrocantalejo@gmail.com \\ ORCID: https://orcid.org/0000-0001-6408-7385 \\ Yvonne Tafelmaier \\ State Office for Cultural Heritage Baden-Wuerttemberg (Alemania) \\ yvonne.tafelmaier@uni-tuebingen.de \\ ORCID: https://orcid.org/0000-0003-0651-4435

\section{Serafín Becerra} \\ Universidad de Cádiz \\ serafinbecerramartin@gmail.com \\ ORCID: https://orcid.org/0000-0002-7060-2854

\section{Eduardo Vijande-Vila \\ Universidad de Cádiz \\ eduardo.vijande@uca.es} \\ ORCID: https://orcid.org/0000-0002-7591-5112
}




\section{Trine Kellberg Nielsen}

Museo Moesgård (Aarhus, Dinamarca)

tkn@moesgaardmuseum.dk

ORCID: https://orcid.org/0000-0001-6481-5661

\section{Miriam Rotgänger}

Universidad de Colonia (Alemania)

kehlm@uni-koeln.de

ORCID: https://orcid.org/0000-0002-4368-2801

\section{Diego Fernández-Sánchez}

Universidad de Cádiz

diego.fernande@uca.es

ORCID: https://orcid.org/0000-0002-7324-049X

\section{Taylor Otto}

Universidad de Colonia (Alemania)

taylor.otto@uni-koeln.de

ORCID: https://orcid.org/0000-0002-1717-7879

\section{Adolfo Moreno-Márquez}

Universidad de Cádiz

amorenom@ual.es

ORCID: https://orcid.org/0000-0001-7061-2131

\section{Mar Espejo}

Ayuntamiento de Ardales-Cueva de Ardales mariadeespejo@gmail.com

ORCID: https://orcid.org/0000-0002-3432-2628

\section{Viviane Bolín}

Neanderthal Museum (Mettmann, Alemania) bolin@neanderthal.de

ORCID: https://orcid.org/0000-0001-8245-4783

\section{Lidia Cabello}

UNED

lidia_cabelloligero@hotmail.com

ORCID: https://orcid.org/0000-0002-6535-2483

\section{Martin Kehl}

Universidad de Coblenza y Landau (Alemania) mkehl@uni-koblenz.de

ORCID: https://orcid.org/0000-0003-4587-5858

\section{Salvador Domínguez-Bella}

Universidad de Cádiz

salvador.dominguez@uca.es

ORCID: https://orcid.org/0000-0003-3892-763X 


\author{
Julia Blumenröther \\ Universidad de Colonia (Alemania) \\ julia.blumenrother@fau.de \\ ORCID: https://orcid.org/0000-0001-8389-2136
}

\title{
Resumen
}

Un equipo interdisciplinar internacional viene realizando nuevas investigaciones en Cueva de Ardales (Ardales, Málaga) y en Sima de las Palomas (Teba, Málaga), enmarcadas en un Proyecto General de Investigación autorizado por la Junta de Andalucía. Presentamos un avance de los datos obtenidos en ambas cavidades en relación a la ocupación vinculada a sociedades neandertales con tecnología de tipo Paleolítico Medio.

Palabras clave: Cueva de Ardales, Sima de las Palomas, Cucarra, Paleolítico Medio, estratigrafía, tecnología lítica.

\section{Abstract}

An international interdisciplinary team is carrying out new research in Cueva de Ardales (Ardales, Málaga) and Sima de las Palomas (Teba, Málaga), framed in a General Research Project authorized by the Junta de Andalucía. We present here preliminary data of Neanderthal occupations and related Middle Palaeolithic technology from both cavities.

Key words: Cueva de Ardales, Sima de las Palomas, Cucarra, Middle Paleolithic, stratigraphy, lithic technology. 


\section{Introducción}

Un equipo hispano-alemán, dirigido por investigadores de la Universidad de Cádiz y del Neanderthal Museum viene realizando, desde el año 2011 nuevas investigaciones en Cueva de Ardales (Ramos et al., Eds., 2014; Ramos y Weniger, 2016) y en Sima de las Palomas de Teba (Weniger y Ramos, Eds., 2014; Weniger y Ramos, 2016) (Figura 1).

Son yacimientos que cuentan con una interesante historiografía (Breuil, 1921; Ramos et al., 1998; Cantalejo et al., 1997, 2003, 2005, 2006; 2014; Medianero et al., 2011) y permiten la posibilidad de ofrecer unas secuencias arqueológicas amplias para la reconstrucción histórica de las ocupaciones de las sociedades cazadoras-recolectoras paleolíticas y tribales neolíticas.

Los primeros resultados fueron de gran interés (Ramos et al., Eds., 2014; Weniger y Ramos, Eds., 2014; Cantalejo et al., 2014) y se solicitó a la Dirección General de Bienes Culturales de la Consejería de Cultura de la Junta de Andalucía un Proyecto General de Investigación -PGI- para profundizar en la secuencia arqueológica de ambas cavidades. Este proyecto ha sido aprobado por dicha institución y lleva por nombre "Las sociedades prehistóricas (del Paleolítico Medio al Neolítico Final) en la Cueva de Ardales y Sima de las Palomas de Teba (Málaga, España). Estudio geoarqueológico, cronológico y medioambiental". Cuenta con la dirección de José Ramos y Gerd-Christian Weniger y está formado por un equipo interdisciplinar con presencia de investigadores de numerosas instituciones científicas. Tenemos la colaboración de los Ayuntamientos de Ardales y Teba.

Vamos a presentar una síntesis de los resultados obtenidos en este Proyecto en
Cueva de Ardales, Cucarra y Sima de las Palomas de Teba.

\section{Cueva de Ardales}

\subsection{Localización geográfica. Marco geológico. La cavidad}

Cueva de Ardales está localizada al sur de la Península Ibérica, en la provincia de Málaga (Figura 1), a menos de cincuenta kilómetros de la costa Mediterránea, en un entorno físico desde el que se controla uno de los pocos pasos naturales que el Sistema Bético permite entre el Mediterráneo y el valle del Guadalquivir. En su entorno inmediato confluyen los cursos fluviales de los ríos Turón, Guadalteba y Guadalhorce, en una zona de grandes cañones kársticos, conocida como Desfiladero de los Gaitanes/Caminito del Rey.

La situación geográfica explica las posibles relaciones costa-interior y E.-O. de los grupos humanos cazadores-recolectores paleolíticos, al estar en medios naturales próximos a la Depresión de Antequera y a la Serranía de Ronda. La zona inmediata constituye un paisaje diversificado de gran complejidad geológica.

Este territorio ofrece una gran variedad de recursos y condiciones ecológicas, donde destacan montañas altas, como Sierras Prieta, Alcaparaín, Ortegícar, entornos de montañas de altura media, actuales campiñas y cursos fluviales. Así mismo ofrece numerosas posibilidades de captación de recursos (cinegéticos, forestales, líticos).

Hemos estudiado los componentes mineralógicos y petrológicos de las áreas geológicas del entorno como posibles zonas de captación (Domínguez-Bella et al., 2001; Domínguez-Bella, 2014 a, 2014 b, Cabello, 2017; Becerra, 2019): materiales silíceos del 
Jurásico-Cretácico (Cordilleras Béticas), rocas ultrabásicas del Macizo de Ronda y otras litologías documentadas en el registro arqueológico.

Hay que indicar también que en este marco geográfico se registra la presencia humana durante todo el Pleistoceno Medio y Superior, tanto en las terrazas de los ríos Turón, Guadalteba y Guadalhorce, como en yacimientos relacionados con grandes abrigos y cuevas (Medianero et al., 2011, 2012; Cantalejo et al., 2014; Cabello, 2017).

La Cueva de Ardales constituye la forma endokárstica más destacada de la Serrezuela, conjunto montañoso localizado entre las localidades de Ardales y Carratraca. Presenta un desarrollo conocido de $1.597 \mathrm{~m}$. proyectados en planta y una morfología alargada en dirección ENE-SW, con unos desniveles máximos de $35 \mathrm{~m}$.

Surgida entre los mármoles y las calizas del Triásico correspondientes a la Unidad BonellaCapellán, la Cueva de Ardales es un sistema de cavidades unidas entre sí, con una única boca de acceso en la actualidad, abierta al $\mathrm{N}$. del Cerro de la Calinoria, a 599 m.s.n.m., en las coordenadas U.T.M. 337.110/4.802.540, aunque durante la Prehistoria tuvo, al menos, una boca más. Es una montaña de calizas y mármoles del Triásico, conocida como Cerro de La Calinoria. La composición de las rocas es mayoritariamente dolomítica o calizodolomítica (Durán y López, 1995), destacando la Gran Sala, la Galería del Arquero, la Sala del Lago, la Galería de los Grabados, la sala de las Manos y El Calvario.

Dentro de la cavidad se observa un cono de sedimentos muy potente, que se utilizó históricamente como zona de acceso, habilitado con unas escaleras a mediados del siglo XIX.

\subsection{Historia de la investigación}

Fue descubierta en 1821 gracias a un terremoto que dejó libre la actual puerta de entrada. Tras ser citada en el Diccionario geográfico-estadístico (Madoz, 1845), la cavidad es adquirida por Trinidad Grund, quien la acondiciona para su visita, abriéndola al público en 1852, como complemento de su negocio termal instalado en la vecina Carratraca, siendo la primera cueva abierta al turismo en España.

El origen de la investigación prehistórica se remonta a 1918, cuando Henri Breuil descubre las primeras figuras grabadas $y$ pintadas, 10 paneles con una veintena de figuras animales (Breuil, 1921). Tras la Guerra Civil el yacimiento se abandona, sin que las instituciones o los investigadores realicen tareas de protección e investigación hasta principios de los años ochenta, cuando el Ayuntamiento de Ardales promueve el primer proyecto de recuperación y estudio de la cavidad (Ramos et al., 1992). A partir de ese momento la cueva se protege, se estudia y se reabre al público, con visitas restringidas (Espejo y Cantalejo, 1987, 1988).

Se han realizado numerosos estudios en las últimas décadas, centrados en el arte prehistórico sobre todo (Sanchidrián, 1998, 2001; Ramos et al., 1998; Cantalejo et al., 2005, 2006). La cueva conserva más de 50 motivos figurativos y cerca de 1000 símbolos pertenecientes al Paleolítico Superior (Cantalejo et al., 2006) y al Paleolítico Medio (Hoffmann et al., 2018). Son figuras de antropomorfos, cérvidos, équidos, cápridos y un pez, grabados en su mayoría con punzones o buriles de sílex. Otras están pintadas en negro, rojo y ocre-amarillo. Junto a estas figuraciones se observan otras en rojo de carácter abstracto, alineaciones de puntos, digitaciones, barras, trazos lineales y curvos o simples manchas (Cantalejo y Espejo, 2013; Cantalejo et al., 1997, 2003, 2006). Uno de los elementos más destacados del conjunto es, precisamente, la representación de manos, de las que se han encontrado dos en negativo y en color negro (Cantalejo et al., 2006: 111-112 y 249-251).

El nuevo PGI mencionado está uniendo las actividades arqueológicas de realización de sondeos estratigráficos, con la aplicación de nuevas tecnologías al arte rupestre paleolítico y a los restos antropológicos, lo que está 
suponiendo una actualización científica, de gran alcance e interés internacional (Hoffmeister et al., 2015; Hoffmann et al., 2018).

\subsection{Actuaciones arqueológicas puntuales y del PGI en Cueva de Ardales}

En los trabajos realizados entre 2011-2014 en el marco de actuaciones arqueológicas puntuales se han realizado (Ramos et al., Eds., 2014; Ramos y Weniger, 2106; Cantalejo et al., 2014):

- Sondeos geoarqueológicos en el exterior.

- Aplicación de georrádar en los exteriores.

- Sondeos geoarqueológicos en el interior.

- Se ha generado una nueva topografía de precisión del exterior e interior para conocer posibles usos por grupos humanos.

- Toma de muestras de productos arqueológicos (exterior).

- Toma de muestras de productos antropológicos (interior).

- Toma de muestras de pólenes y estudio de fauna (interior).

- Toma de muestra de carbones (interior).

- Aplicación de barridos láser al arte rupestre.

- Análisis de pigmentos.

- Análisis arqueométrico de productos arqueológicos.

- Análisis con tecnología Raman de productos líticos, lámparas, carbones y arte.

- Dataciones absolutas (TL, OSL, $\mathrm{U} /$ th, C14). Los objetivos de estas actividades realizadas se enmarcan en la necesidad de avanzar en estudios geoarqueológicos.
Estos trabajos han generado una gran información y un auténtico relanzamiento de los trabajos de investigación que veníamos desarrollando desde los años 80 del siglo pasado (Ramos et al., 1998; Cantalejo et al., 2006).

El nuevo PGI desarrollado entre $2015-2018$ ha permitido conformar un proyecto internacional e interdisciplinar, donde se está obteniendo gran información del contexto medioambiental, con sondeos, estudios estratigráficos, cronológicos, Arqueozoología, Arqueobotánica, Arqueometría, Arqueología científica... (Cantalejo et al., 2014; Hoffmeister et al., 2015; Ramos y Weniger, 2016, Hoffmann et al., 2018; Ramos et al., 2019; Cantillo et al., e.p.).

Se trata por tanto de un proyecto arqueológico que pretende obtener información de base de estudios de tipo histórico y social para profundizar en la movilidad, en las prácticas sociales y económicas, así como en los modos de vida de las sociedades cazadoras-recolectoras paleolíticas y tribales comunitarias neolíticas que frecuentaron la cavidad. Representa un trabajo en equipo interdisciplinar donde participan más de 50 investigadores pertenecientes a 28 instituciones. Indicamos aquí algunas de las líneas de trabajo:

- En las campañas de 2015, 2016, 2017 y 2018 se han hecho sondeos estratigráficos en las zonas 2, 3 y 5 (Figura 2). Se han podido datar diversos elementos orgánicos y se ha obtenido un interesante cuadro cronoestratigráfico (Ramos y Weniger, 2016).

- Estudios de micromorfología de suelos, en realización por Martin Kehl (University of Cologne).

- Los estudios de micromamíferos están siendo realizados por Juan Rofes (Muséum National d'Histoire Naturelle de París).

- Los anfibios y reptiles son analizados por Salvador Bailón 
(Muséum National d'Histoire Naturelle de París).

- El estudio de macromamíferos está a cargo de José Antonio Riquelme y Alejandro Beltrán (Universidad de Córdoba).

- El apartado de arqueomalacología es realizado por Juan Jesús Cantillo (Universidad de Cádiz). Y la funcionalidad de las conchas por David Cuenca (Universidad de Cantabria).

- Los estudios arqueobotánicos tienen la responsabilidad de Paloma Uzquiano (UNED).

- Los estudios polínicos son realizados por Blanca Ruiz Zapata y María José Gil (Universidad de Alcalá de Henares).

- Pablo Ramos García (Universidad de Granada) ha estudiado los restos humanos dentarios estratificados de las zonas 2 y 5 .

- Alfonso Palomo y Víctor Manuel Smith (Universidad de Málaga) han analizado los restos humanos documentados en diversas zonas de la cavidad.

- El estudio de las materias primas líticas viene siendo realizado por Salvador Domínguez-Bella (Universidad de Cádiz), Serafín Becerra y Lidia Cabello.

- El análisis tecnológico de los productos líticos tallados ha corrido a cargo de José Ramos, Gerd C.-Weniger, Yvone Tafelmaier, Antonio Barrena, Sergio Almisas, Eduardo Vijande, Serafín Becerra, Pedro Cantalejo, Lidia Cabello, Serafín Becerra, Diego Fernández y María del Mar Espejo.
- Ignacio Clemente (CSIC Barcelona) estudia la funcionalidad de los productos líticos.

- El estudio del ocre está siendo analizado por África Pitarch (Pacea. Université de Bordeaux).

- La aplicación de las series del uranio al arte rupestre de estilo paleolítico han corrido a cargo de Dirk Hoffmann (Max Planck Institute for Evolutionary Anthropology, Leipzig), Marcos García Díaz (Universidad Isabel I de Burgos), Joao Zilhao (ICREA. Universidad de Barcelona), Paul Pettitt (Durhan University), Alistair Pike (Southantomp University), Chris Standisch (Southantomp University), con la colaboración de Pedro Cantalejo, Gerd Weniger y José Ramos.

- El estudio de documentación de las manos paleolíticas ha sido realizado por Hipólito Collado (Junta de Extremadura), Julio Angás y Manuel Bea (Universidad de Zaragoza).

- En las Galerías Altas se han efectuado muestreos superficiales de posibles lámparas y la datación de una mandíbula humana depositada en el Museo de Ardales.

- En el momento actual se está preparando toda la base de datos y la memoria de las campañas 2017, 2018; así como la memoria final del PGI.

\subsection{Nuevas excavaciones. Cueva de Ardales Zona 3}

2.4.1 Excavación, estratigrafía y cronologías

En el marco de este Proyecto se han realizado sondeos en las zonas 2, 3 y 5 de la cavidad (Figura 2). En resumen podemos indicar la presencia de una completa secuencia de los resultados arqueológicos que tienen relación 
con los motivos del arte. Este proyecto aúna las tareas arqueológicas de realización de sondeos estratigráficos, con la aplicación de nuevas tecnologías al Arte Rupestre Paleolítico y a los restos antropológicos, lo que está suponiendo una actualización científica, cuyos resultados están alcanzando un interés internacional (Hoffmeister et al., 2015; Hoffman et al., 2018). Hay una amplia serie de dataciones paleolíticas en las zonas 2,3 y 5 , que se asocian con productos líticos tallados vinculados a Modo 3-Paleolítico Medio en la zona 3 y de varias etapas del Modo 4Paleolítico Superior en las zonas 2 y 5 (Ramos y Weniger, 2016).

\subsubsection{Productos arqueológicos}

Los productos líticos documentados son limitados todavía, pero muy uniformes y claros tecnológicamente. Se ha controlado un ejemplar de BN1G-Núcleo, con talla periférica de aspecto unipolar. Hay BP-Lascas de técnica levallois y algún ejemplar de BN2G-Raedera. Están realizados sobre todo en sílex.

En general, se puede decir que el conjunto estudiado de la zona 3 está formado por diferentes materias primas, con presencia entre otros, de sílex de tipo Turón y sílex masivos, que son básicamente de origen local, documentadas en las unidades geológicas del entorno del yacimiento. Hay que indicar también la presencia de ocre en los estratos vinculados a tecnología de Paleolítico Medio.

\section{Cucarra}

\subsection{Situación geográfica y contexto geológico}

El yacimiento está ubicado a unos $50 \mathrm{~m}$. al E. de la Cueva de Ardales, junto a una fuente natural (Figura 3). Se accede cruzando la carretera A-357 desde la localidad de Ardales en dirección a la Cueva. Este entorno presenta materiales pertenecientes a las Zonas Internas de las Cordilleras Béticas, situados al límite de la Zona Externa. Se ubica en el afloramiento de materiales carbonaticos que se localiza entre las localidades de Ardales y Carratraca (la Serrezuela), en concreto en la Unidad de Yunquera, dentro del Complejo Alpujárride, con presencia de dolomías y calizasdolomíticas (Cabello, 2017: 105).

El enclave tiene un origen de abandono antrópico de los productos líticos, que están distribuidos en los depósitos arcillosos en la ladera ubicada bajo la entrada de la Cueva de Ardales. En la zona se han plantado almendros y olivos (Cabello, 2017: 105).

\subsection{Historia de la investigación}

El yacimiento fue localizado por Mar Espejo y Pedro Cantalejo en los años 80 en el marco del Proyecto de prospecciones del río Turón y durante los estudios en Cueva de Ardales. Se ha continuado el control de los materiales durante los trabajos de 2011-2014 en la Actuación Arqueológica Puntual, desarrollada en Cueva de Ardales.

El estudio lítico que presentamos se realiza sobre el material recogido durante las prospecciones de los años 80 y los muestreos geoarqueológicos llevados a cabo en las intervenciones arqueológicas entre 20112014 (Ramos et al., Eds., 2014). Posteriormente ha sido analizado por Lidia Cabello en su Tesis Doctoral, realizando un estudio de materias primas, análisis tecnológico y tipológico (Cabello, 2017: 105 y ss.).

\subsection{Productos líticos}

Entre las materias primas predominan sílex masivo gris o beige con pátina amarillenta o blanca. En menor medida sílex poroso de tonalidades grises y con pátinas amarillentas y blancas, radiolaritas y cuarcitas.

Se han documentado ejemplares de BN1GNúcleos, BP-Lascas y BN2G-Productos retocados. Entre los BN1G-Nucleos predominan los centrípetos multipolares.

Entre las BP-Lascas, destacan especialmente lascas, con alguna presencia mucho menor de alguna lámina. Algunos ejemplares cuentan con pátina de color amarillo, con rodamiento medio y cúpulas de termoalteración. Están documentados ejemplares de BP-D-Lascas de 
descortezado,

BP-Lascas de semidescortezado, BP-I-Lascas internas y predominan BP-L-Lascas levallois (Figura 4). Entre los talones dominan los facetados convexos con bulbo marcado. En las caras dorsales predominan las no corticales.

Entre los productos retocados predominan ejemplares de BN2G-Raederas (Figura 5), con presencia de R21-Raederas laterales y R23Raedera latero-transversal, realizadas básicamente sobre lascas levallois. Hay registro de algunos ejemplares DDenticulados y un G-Raspador (Cabello, 2017: 105 y ss.).

\section{Sima de las Palomas de Teba}

\subsection{Situación geográfica y contexto geológico}

El Complejo Kárstico de Las Palomas (Figura 6) se sitúa en la parte oriental de la sierrade Peñarrubia dentro del término municipal de Teba, sobre el paraje del Tajo del Molino, cortado que separa esta sierra de la de Teba y por el que discurre el río de La Venta. El yacimiento se encuentra a $3,5 \mathrm{~km}$. del casco urbano de la localidad, accediéndose desde la carretera C-341.

El complejo kárstico tiene un desarrollo próximo a los $150 \mathrm{~m}$. de longitud que va desde los 470 hasta los 430 m.s.n.m. (Medianero et al., 2011), punto en el que se precipita más de treinta metros sobre el río de la Venta. Este curso fluvial es el principal elemento que genera la discontinuidad en la Sierra de Peñarrubia-Teba, erosionando la roca caliza, sus aguas que recorren este estrecho desfiladero y vierten al río Guadalteba. En términos geológicos, este paraje se inserta en un relieve kárstico originado sobre las calizas jurásicas del Penibético/Subbético interno (Cruz-Sanjulián, 1990).

El enclave se sitúa en el pie de monte, controlando excelentes tierras para el cultivo, las cuales en la actualidad se dedican a la agricultura de secano. La sierra ofrecía a las sociedades prehistóricas acceder a un innumerable abanico de posibilidades cinegéticas y forestales, mientras que el río Guadalteba, a escasos 3,5 km, reportaba recursos acuáticos. En la base de la sierra de Peñarrubia-Teba se encuentra el manantial de Torrox, el cual favorece la implantación de huertas en las fértiles tierras que se disponen en su entorno.

Las materias primas líticas (Cabello, 2017: 105-106; Becerra, 2019) más próximas son los afloramientos de sílex y radiolaritas de la Sierra de Cañete, así como las radiolaritas pertenecientes a unidades insertas en el complejo de Campo de Gibraltar situadas al oeste del yacimiento (Cruz-Sanjulián, 1990). Las terrazas del Guadalteba también debieron de constituir lugares de aprovisionamiento lítico, así como la franja geológica con sílex que va de Campillos hacia Almargen.

Desde el yacimiento se controla la vía que une las tierras de Antequera y la Serranía de Ronda, este pasillo natural es el denominado Surco Intrabético.

\subsection{Historia de la investigación}

A comienzos de la década de los 70 del siglo $\mathrm{XX}$, de la mano del maestro Pedro Pérez se realizaron en la Cueva de las Palomas una serie de intervenciones de carácter no científico que pusieron de manifiesto la relevancia arqueológica del enclave (Baldomero, 1978). Puesto en conocimiento del Departamento de Prehistoria y Arqueología de la Universidad de Málaga, se procedió a desarrollar una serie de excavaciones arqueológicas entre 1975 y 1977 con el permiso de la Dirección General de Patrimonio Artístico y Cultural. Derivadas de esta serie de intervenciones son diferentes publicaciones (Aguado y Baldomero, 1979; Ferrer y Fernández, 1988; Ferrer y Márques, 1978) y Memorias de Licenciatura (Leiva, 1977; Fontao, 1986; Fernández, 1986).

Tras esta etapa de investigación, las publicaciones sobre el yacimiento disminuirán, quedando reducidas a síntesis y recopilaciones bibliográficas en estudios territoriales de la zona y la provincia (Cortés et al., 1993; Recio, 1993; Morgado, 1995; 
Rodríguez y Márquez, 2003). Pero a partir del año 2002, la situación va a cambiar, ya que con la coordinación de Pedro Cantalejo, desde el Consorcio Guadalteba, en el marco del programa de Escuelas Taller y con dirección de Javier Medianero, se llevará a cabo la limpieza y "puesta en valor" del yacimiento (Medianero, 2007, 2010).

La investigación estuvo centrada inicialmente en la Cueva de las Palomas, hasta que se inician una serie de exploraciones en la Sima de Las Palomas, denominada por sus descubridores como Sima del Sílex (Morgado, 1995). Esta cavidad, se sitúa a escasos metros de la boca de entrada de la cueva principal, conformando otro sector de enorme potencial arqueológico en el denominado Complejo Kárstico de Las Palomas (Medianero et al., 2011, 2012). En este sentido, en el año 2011 se desarrolló una actividad arqueológica puntual en el yacimiento dirigida por GerdChristian Weniger (Stiftung Neanderthal Museum) y con la codirección de José Ramos (Universidad de Cádiz). Los interesantes resultados que reportó esta intervención (Weniger y Ramos, 2014), motivaron que el equipo hispano-alemán iniciará en 2015 nuevos trabajos arqueológicos en el marco del PGI indicado, en paralelo a las actividades indicadas realizadas en Cueva de Ardales.

\subsection{Estratigrafía. Avance de los productos arqueológicos líticos de los niveles 9-10 de Sima de las Palomas}

La Sima de las Palomas corresponde a una cavidad que tiene actualmente una profundidad de unos $7 \mathrm{~m}$. Fue objeto de una extracción de sedimentos descontrolada, desconociendo su origen por el momento, que se realizó sobre los sedimentos de un antiguo abrigo o galería que se comunicaba con la cueva, aunque actualmente la presencia de bloques impide enlazar ambos yacimientos.

El registro arqueológico documentado constata una intensa ocupación (Figura 7) del Paleolítico Medio, así como la presencia de comunidades del Paleolítico Superior
(Weniger y Ramos, Ed., 2014. Kehl, et al., 2013, 2016).

De los 10 estratos arqueológicos diferenciados, las unidades 9-10 son las que presentan una mayor densidad de productos líticos, óseos y carbones. Se trata de niveles con matriz de arcillas limosas de color marrón rojizo. A mitad del nivel 10 aparecen evidencias de combustión que parecen corresponderse con hogares. Las dataciones por radiocarbono y por OSL del nivel 10 establecen una horquilla temporal entre los 50-60 Ka. BP (Kehl et al., 2016).

Este momento de ocupación del Paleolítico Medio se caracteriza por un bosque regional de Pinus y Juniperus. Sin embargo, este bosque regional da paso a una mayor presencia de un bosque local constituido por especies perennifolias o mediterráneas y a un proceso de retroceso de la masa forestal, que a techo del estrato 9 evidencia una pérdida de humedad y un descenso de las temperaturas. Estas condiciones climáticas parece que dan lugar a un paisaje con mayor presencia de espacios abiertos (Ruiz Zapata y Gil, 2014). Este territorio estaría ocupado por una macrofauna de tamaño medio, como el ciervo, la cabra montés o el jabalí, así como otra de mayor porte como el caballo o el uro (Espigares et al., 2014).

El análisis del perfil estratigráfico (trabajos 2011-2014) ha permitido documentar en los estratos 9-10, 309 productos líticos (Figura 8). Del estudio realizado (Ramos et al., 2014) se desprende que:

Hay un claro predominio de los restos de talla sobre los productos retocados, concretamente el 94,50 (292 ejemplares) de la industria analizada son restos de talla, y solo el 5,50\% (17 ejemplares) son productos retocados.

De estas unidades se recuperaron 11 ejemplares de BN1G, de los cuales 10 son BN1G-Centrípeto multipolar y 1 BN1GBipolar.

Los ejemplares de BP-Lascas y/o láminas contabilizados fueron 191. Existe un 
predominio de las BP-Internas (129 ejemplares), seguido de un alto porcentaje (26,18\%) de BP-Levallois (50 ejemplares), y una representación escasa de BP del inicio de talla y crestas $(4,74 \%$ y $0,52 \%$ respectivamente). Entre los productos recuperados se documentó una BP-Punta levallois.

La importante presencia (90 ejemplares) de ORT-Otros productos de talla pone de manifiesto la existencia de procesos de desbaste y talla.

De BN2G-Productos retocados se han analizado 17 ejemplares. El grupo más numeroso es el de las muescas y denticulados, con 9 ejemplares; el resto de BN2G se corresponden con raederas planas, en concreto 1 ejemplar de raedera plana con retoque marginal lateral, y 7 de raederas planas con retoques profundos laterales.

Los datos planteados sugieren la presencia de procesos de talla en el propio asentamiento, donde existe una gran sintonía en los rasgos técnicos entre las BP y las BN2G. Es una tecnología vinculada al Modo 3-Paleolítico Medio, donde se lleva a cabo una producción de BP-Lascas para una posterior transformación o bien para el empleo directo en actividades productivas como el procesado de animales y recursos vegetales leñosos (Clemente, 2014).

Los niveles de ocupación 9-10 vinculados al Paleolítico Medio, evidencian una intensa y reiterada ocupación del espacio, la cual se ve interrumpida probablemente por el desprendimiento del techo de la cavidad (unidad 8). Con posterioridad, grupos humanos con tecnología del Modo 3 volverán a visitar el enclave de manera esporádica (unidad 6) tras una etapa de abandono (unidad 7).

\section{Valoración de datos arqueológicos vinculados a Paleolítico Medio en Cueva de Ardales, Cucarra y Sima de las Palomas de Teba}

Los últimos trabajos desarrollados en Cueva de Ardales y en Sima de las Palomas de Teba están resultando de gran interés.

Los resultados obtenidos hasta la fecha demuestran que la Cueva de Ardales es un yacimiento que cuenta con una cronología bastante amplia. Hay diversas fases de ocupación y fases de hiatus temporales con vacíos de ocupación. Los sondeos son de superficies limitadas y la morfologíaestructura microespacial multivariada de la cavidad con zonas muy pendientes y zonas planas hacen todavía difícil la interpretación completa de la ocupación en el espacio, en el momento actual de la investigación. De todos modos, ofrecen una destacada secuencia con registros de Paleolítico Medio y Paleolítico Superior.

En concreto en Cueva de Ardales, en la zona 3 $\left(4 \mathrm{~m}^{2}\right)$, que forma parte de la Sala del Saco, se ha documentado la presencia de ocupaciones estratificadas de Paleolítico Medio-Modo 3, con cronologías inferiores a $40 \mathrm{Ka}$. Esta zona es poco inclinada y bastante útil para una ocupación (Ramos et al., Eds., 2014). En las últimas campañas se han documentado productos líticos estratificados de Paleolítico Medio-Modo 3 (BN1G-Núcleos centrípetos, BP-Lascas levallois y BN2G-Raederas).

Hemos aportado datos del yacimiento al aire libre de Cucarra, vinculado a las ocupaciones de Paleolítico Medio de Cueva de Ardales, que representa un campamento al aire libre, con una tecnología muy uniforme y de gran relación tecnológica con la documentada en los niveles de dicha época de la zona 3 de Cueva de Ardales.

En Sima de las Palomas de Teba, en un entorno muy cercano de $15 \mathrm{kms}$., que presenta también una secuencia arqueológica de gran interés se han documentado ocupaciones de etapas históricas medievales, así como a sociedades prehistóricas: 
Calcolítico, Neolítico, Paleolítico SuperiorModo 4 y Paleolítico Medio-Modo 3 (Weniger y Ramos, Eds., 2014; Kehl et al., 2013, 2016). En el perfil estratigráfico documentado en los trabajos realizados entre 2011 y 2014, en los niveles, 9 y 10, la tecnología lítica es muy clara, formada por BN1G-Núcleos centrípetos, BPLascas levallois y BN2G-Raederas y puntas variadas, junto a muescas y denticulados. Las dataciones absolutas son muy coherentes con las obtenidas en Cueva de Ardales y se sitúan entre 50-60 Ka BP (Kehl et al., 2016).

Hemos elaborado un estudio teórico del área de explotación-Site Catchment- de 4 horas de movilidad desde Cueva de Ardales y Sima de las Palomas. Es muy significativa la superposición de dichas áreas. Es muy posible el uso de estos territorios inmediatos por la vinculación ecológica de la fauna y la procedencia de las materias primas. Básicamente destacan los usos de sílex masivos, sílex de tipo Turón y de tipo Azulejo en ambas cavidades y junto a ellos la destacada presencia de radiolaritas rojas y verdes en Sima de las Palomas procedentes de áreas cercanas (Cabello 2017: 105-106; Becerra, 2019).

Como hipótesis de trabajo estamos indicando la existencia de campamentos en Cucarra y Sima de las Palomas, con cierta estabilidad y ocupación recurrente, al menos en Sima de las Palomas. En este sentido Cucarra sería el asentamiento al aire libre y Cueva de Ardales, sería un refugio, asociado al desarrollo de actividades especiales y de representación de manifestaciones gráficas.

Resulta de interés la asociación de las cronologías aportadas por Cueva de Ardales, en relación a la coherencia de los productos líticos de Zona 3, con la presencia de ocre, así como de fechas análogas en las dataciones de U/Th sobre motivos pintados (Hoffman et al., 2018). Todo ello se asociaría con la ocupación de sociedades neandertales en la cavidad.

En el contexto regional, hay que poner en relación estos novedosos resultados con la información disponible de las destacadas ocupaciones de Gibraltar (Finlayson et al.,
2006), Bajondillo (Cortés, 2007), Zafarraya (Barroso y De Lumley, Dir., 2006), ), Carihuela (Fernández et al., 2007), Higueral de Valleja (Jennings et al., 2009), Higueral de Motillas (Giles et al., 1998), entre otras cavidades excavadas, para entender la importante presencia en el registro territorial de ocupaciones neandertales en el sur de Iberia (Vallespí, 1986; Cortés et al., 2011-2012; Wood et al., 2013; Weniger y Ramos, Eds., 2014).

La excavación en Sima de las Palomas, permite en el desarrollo del PGI y en asociación de los datos de Cueva de Ardales y de Cucarra incidir en el territorio de la zona de los valles de los ríos Turón, Guadalhorce y Guadalteba (Medianero et al., 2011, 2012; Cabello, 2017) y seguir profundizando en el conocimiento de los modos de vida de las comunidades cazadoras-recolectoras neandertales que habitaron la zona actual de los valles del Turón y Guadalteba.

\section{Anexos}

Figura 1. Localización geográfica de Cueva de Ardales y Sima de las Palomas de Teba

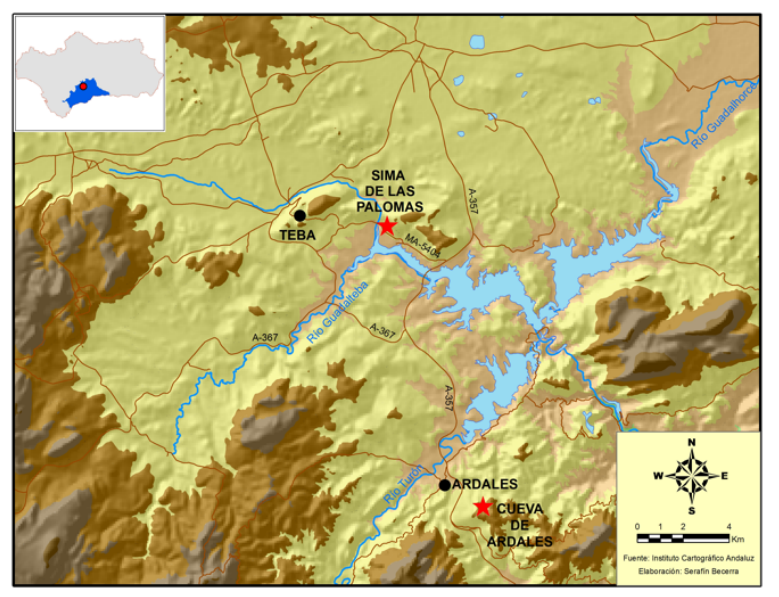


Figura 2. Cueva de Ardales. Zonas arqueológicas excavadas.20112018.

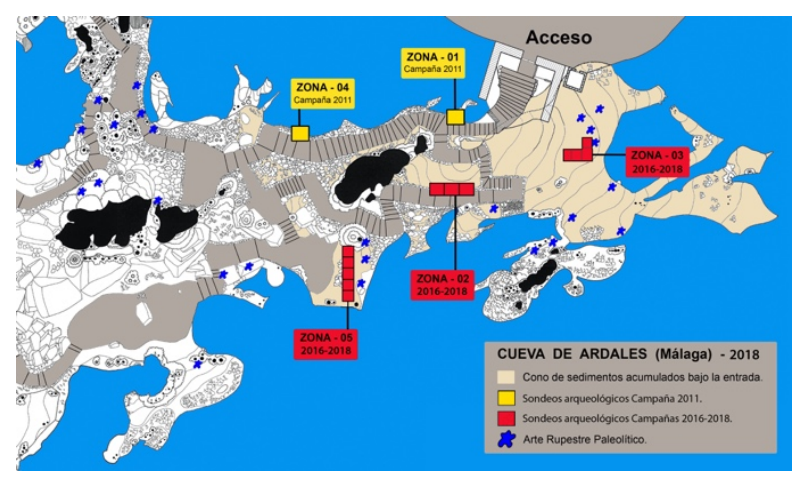

Figura 3. Vista de Cueva de Ardales y del asentamiento al aire libre de Cucarra.

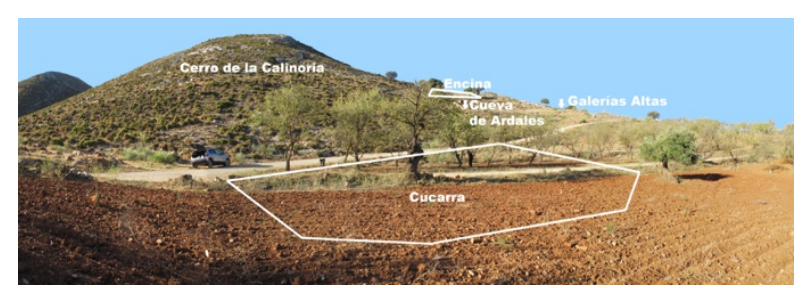

Figura 4. Cucarra. BP-Lascas.

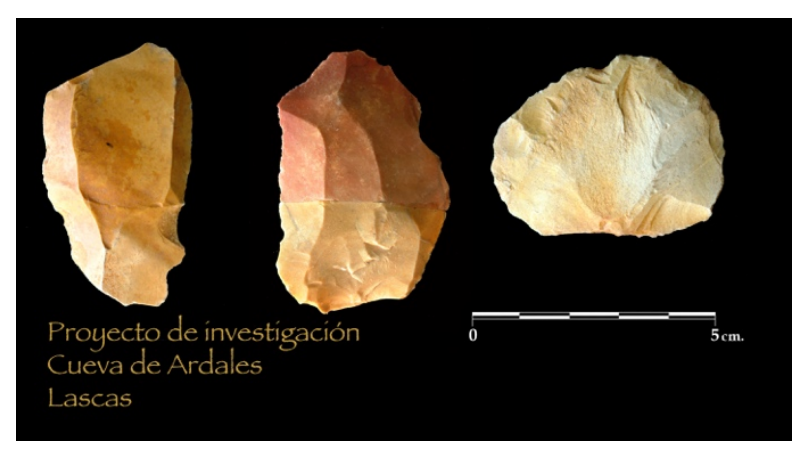

Figura 5. Cucarra. BN2G-Raederas.

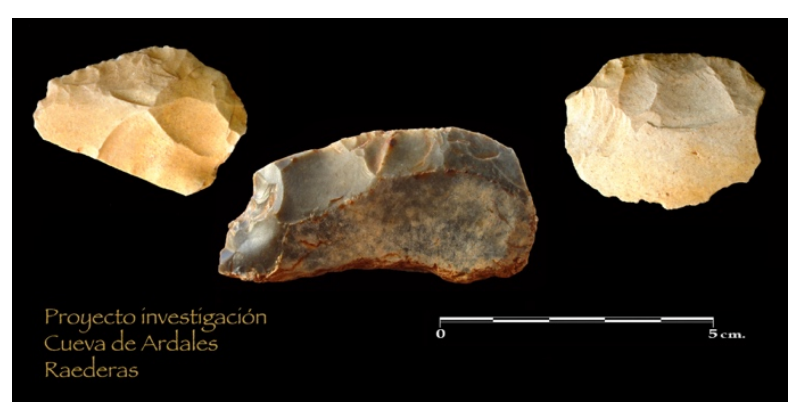

Figura 6. Sima de las Palomas. Perfil complejo kárstico.

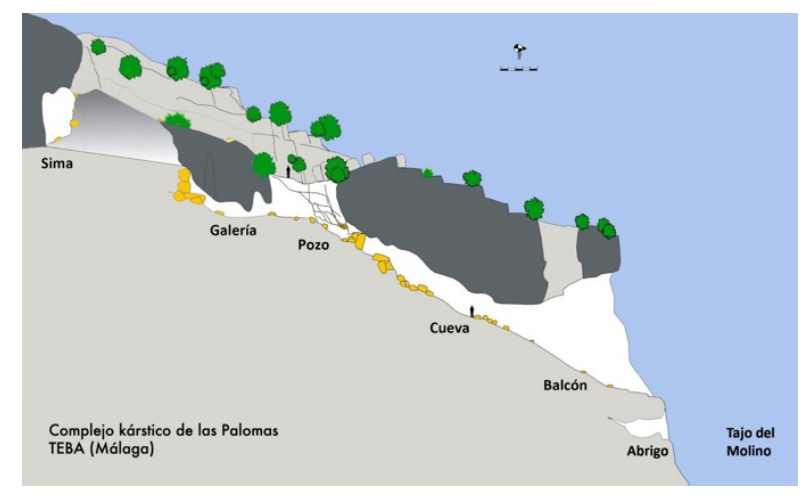

Figura 7. Sima de las Palomas. Perfil estratigráfico.

\section{PERFIL ESTRATIGRÁFICO DE LA SIMA DE LAS PALOMAS}

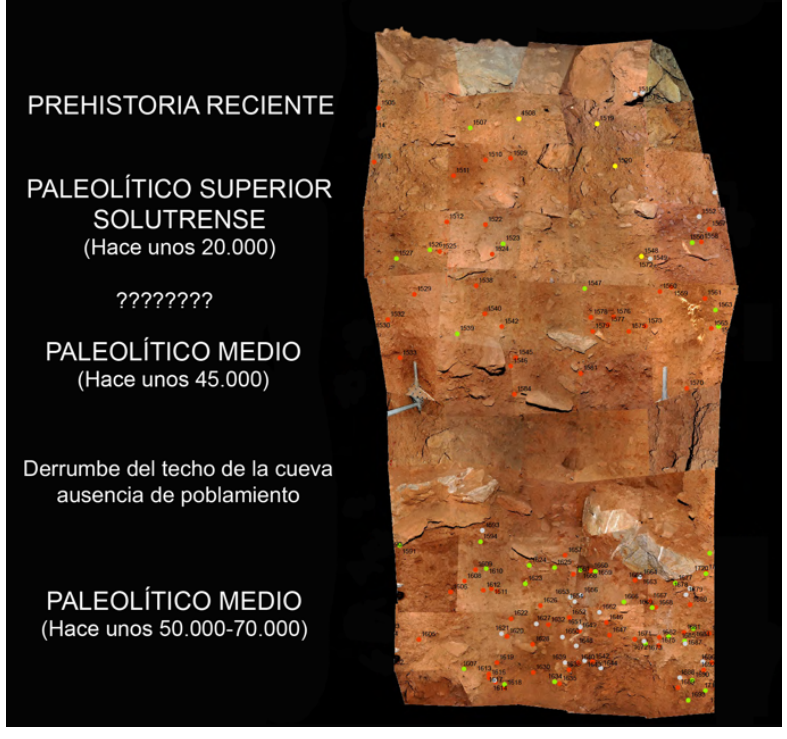

Figura 8. BN1G-Núcleos, BP-Lascas (Kehl et al., 2016).

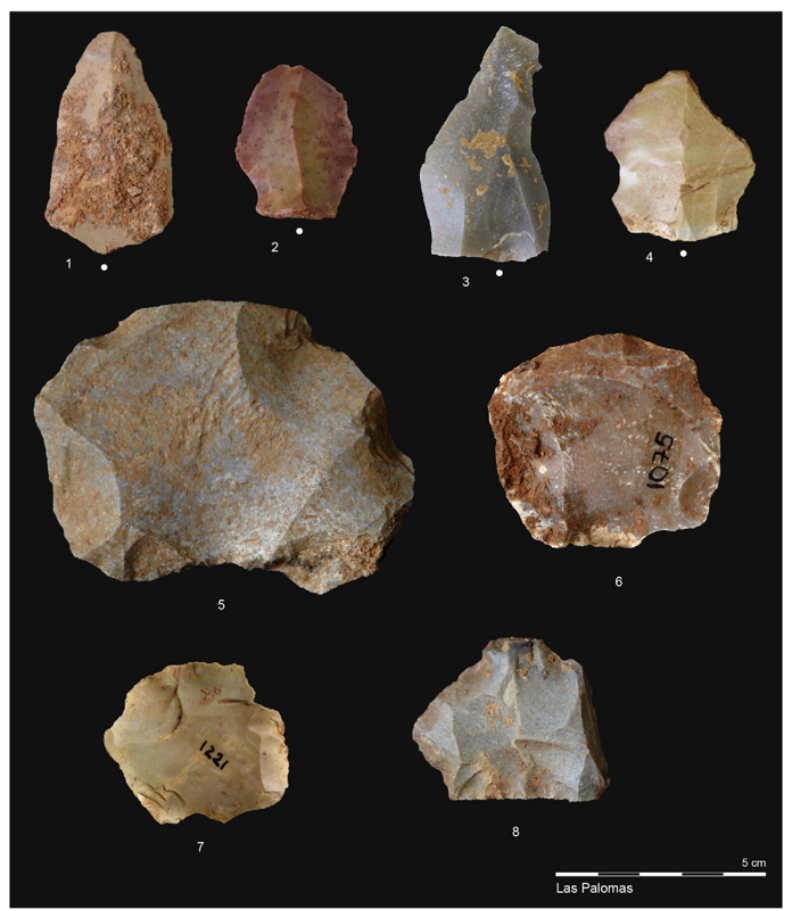




\section{Bibliografía}

AGUADO MANCHA, Teresa y BALDOMERO NAVARRO, Ana, "Estudio de los materiales de superficie de la Cueva de las Palomas. Teba (Málaga)", en Mainake I, 1979, 29-59.

BALDOMERO NAVARRO, Ana, "Noticias del hallazgo de un vaso eneolítico", en Baetica 1, 1978, 167-170.

BARROSO RUIZ, Cecilio y DE LUMLEY, Henry, Dir., La Grotte du Boquete de Zafarraya. Málaga, Andalousie, Sevilla, Consejería de Cultura. Junta de Andalucía, 2006.

BECERRA MARTÍN, Serafín, EI aprovisionamiento del silex durante la Prehistoria Reciente en la Comarca del Guadalteba (Málaga). Un análisis de la arqueometría y la tecnología lítica, Oxford, BAR International Series, 2019.

BREUIL, Henri, "Nouvelles cavernes ornées Paléolithiques dans la province de Málaga", en L'Anthropologie, 31, 1921, 239-253.

CABELLO LIGERO, Lidia, La ocupación humana del territorio de la comarca del río Guadalteba (Málaga) durante el Pleistoceno, Oxford, Archaeopress Archaeology, 2017.

CANTALEJO DUARTE, Pedro y ESPEJO HERRERÍAS, María del Mar, "Cueva de Ardales (Málaga, España). Patrimonio prehistórico en el sur de la Península Ibérica", en PASTOORS, Andreas y AUFFERMANN, Bärbel, Eds., Pleistocene Foragers on the Iberian Península. Their Culture an Environment, Homenaje a Gerd Christian Weniger, Mettman, Neanderthal Museum Wissenschaft, 2013, pp. 101-117.

CANTALEJO DUARTE, Pedro, ESPEJO HERRERÍAS, María del Mar y RAMOS MUÑOZ, José, Cueva de Ardales. Guía Arqueológica, Málaga, Ayuntamiento de Ardales, 1997.

CANTALEJO DUARTE, Pedro, MAURA MIJARES, Rafael, ESPEJO HERRERÍAS, María del Mar, RAMOS MUÑOZ, José, MEDIANERO SOTO, Javier, ARANDA CRUCES, Antonio, MORA, José; BECERRA MARTÍN, Manuel y CASTAÑEDA FERNÁNDEZ, Vicente, "La Cueva de Ardales. Primeras agregaciones gráficas paleolíticas en la Sala de las Estrellas", en Mainake XXV, 2003, 231-248.

CANTALEJO DUARTE, Pedro, MAURA MIJARES, Rafael, ESPEJO HERRERÍAS, María del Mar, RAMOS MUÑOZ, José, MEDIANERO SOTO, Javier Y ARANDA CRUCES, Antonio, "Evidencias de frecuentación prehistórica registradas en la cueva de Ardales (Málaga)", en Actas del IV Simposio de Prehistoria Cueva de Nerja. La Cuenca mediterránea durante el Paleolítico superior, Málaga, Málaga, Patronato de la Cueva de Nerja, 2005, 352364.

CANTALEJO DUARTE, Pedro, MAURA MIJARES, Rafael, ESPEJO HERRERÍAS, María del Mar, RAMOS MUÑOZ, José, MEDIANERO SOTO, Javier y ARANDA CRUCES, Antonio, La Cueva de Ardales: arte prehistórico y ocupación en el Paleolítico Superior. Estudios 1985-2005, Málaga, Centro de Ediciones de la Diputación Provincial de Málaga, 2006.

CANTALEJO DUARTE, Pedro, RAMOS MUÑOZ, José, WENIGER, Gerd-Christian, KEHL, Martin, ESPEJO, Maria del Mar, "Cueva de Ardales, Province of Málaga", en SALA (Ed.), Pleistocene and Holocene hunter-gatherers in Iberia and the Gibraltar Strait: The current Archaeological Record, Burgos, Universidad de Burgos y Fundación Atapuerca, 2014, 426429.

CORTÉS SÁNCHEZ, Miguel, Cueva de Bajondillo (Torremolinos). Secuencia cronocultural y paleoambiental del Cuaternario reciente en la Bahía de Málaga. Málaga. Centro de Ediciones de la Diputación Provincial de Málaga, 2007.

CANTILLO DUARTE, Juan Jesús, WENIGER, Gerd-Christian, RAMOS MUÑOZ, José, CANTALEJO, Pedro, TAFELMAIER, Yvonne, BECERRA, Serafín, BOLÍN, Viviane, ESPEJO, María del Mar, CABELLO, Lidia, FERNÁNDEZSÁNCHEZ, Diego, MORENO-MÁRQUEZ, Adolfo, VIJANDE VILA, Eduardo, BARRENA TOCINO, Antonio, OTTO, Taylor, ALMISAS CRUZ, Sergio, DOMínGUEZ-BELLA, Salvador, PASTOORS, Andreas, "Moluscos marinos y 
fluviales en la Cueva de Ardales y Sima de las Palomas de Teba, Málaga. Primeros resultados de sociedades cazadorasrecolectoras asociadas a tecnología del Paleolítico Superior", en Actas 5RCAPI, Faro, En prensa.

CLEMENTE CONTE, Ignacio, "Estudio preliminar del análisis macro y macroscópico de una selección de piezas líticas de Sima de las Palomas", en WENIGER y RAMOS, Eds., Sima de las Palomas de Teba 2011-2014. Intervenciones arqueológicas, Málaga, Ediciones Pinsapar, 2014, 179-190.

CORTÉS SÁNCHEZ, Miguel, MUÑOZ VIVAS, Victoria Eugenia, SANCHIDRIÁN TORTI, José Luis y SIMON VALLEJO, María Dolores, Corpus historiográfico de Prehistoria Malagueña (1847-1992), Málaga, Edinford, 1993.

CORTÉS SÁNCHEZ, María Dolores, JIMÉNEZESPEJO, Francisco Javier, RODRÍGUEZ VIDAL, Joaquín, MORALES MUÑIZ, Arturo y SIMÓN VALLEJO, María Dolores, "Primeras ocupaciones humanas y fase antigua del Paleolítico Medio meridional ibérico en la Bahía de Málaga", en Mainake XXXIII, 20112012, 63-82.

CRUZ-SANJULIAN, J. JAVIER, Mapa geológico de España. Teba. Hoja 1037, Madrid, Instituto Geológico y Minero de España, Madrid, 1990.

DOMÍNGUEZ-BELLA, Salvador, RAMOS MUÑOZ, José, CANTALEJO DUARTE, Pedro, ESPEJO HERRERÍAS, María del Mar, CASTAÑEDA FERNÁNDEZ, Vicente y DURANTE MACÍAS, Ana, "Lithic resources in the prehistoric societies of the III-II millenniums B.C. in the Río Turón Valley (Ardales, Málaga, Spain), en Slovak Geological Magazine 7, 4, 2001, 319-328.

DOMÍNGUEZ-BELLA, Salvador., RAMOS MUÑOZ, José, WENIGER, Gerd-Christian, CANTALEJO DUARTE, Pedro, ESPEJO HERRERÍAS, María del Mar, MEDIANERO SOTO, Javier, BECERRA MARTÍN, Serafín, CABELLO LIGERO, Lidia, "Materias primas líticas. Estudio arqueomineralógicos y geoarqueológicos", en RAMOS MUÑOZ, WENIGER, CANTALEJO DUARTE, ESPEJO
HERRERÍAS, Eds., Cueva de Ardales 20112014. Intervenciones arqueológicas, Málaga, Ediciones Pinsapar, 2014, 145-150.

DURÁN VALSERO, Juan José y LÓPEZMARTÍNEZ, Jerónimo, 1995: “El karst de la Serrezuela y la Cueva de Ardales: aspectos geológicos, geomorfológicos e hidrogeológicos", en CANTALEJO, DURÁN, ESPEJO, LÓPEZ, MARTÍN, RAMÍREZ, RAMOS, RECIO, Eds., Geología y Arqueología prehistórica de Ardales, Málaga, Ayuntamiento de Ardales, 1995, 47-54. Málaga.

ESPEJO HERRERÍAS, María del Mar y CANTALEJO DUARTE, Pedro, "Nuevas aportaciones al Corpus artístico paleolítico del extremo occidental del Mediterráneo", en RIPOLL, Ed., Actas del Congreso Internacional del Estrecho de Gibraltar, vol. I, Madrid, UNED, 1987, 131-146.

ESPEJO HERRERÍAS, María del Mar y CANTALEJO DUARTE, Pedro, "Cueva de Ardales, yacimiento recuperado", en Revista de Arqueología 84, 1988, 14-24.

ESPIGARES ORTIZ, María Patrocinio, M., ROSMONTOYA, Sergio, RIQUELME CANTAL, José Antonio, PALMQVIST BARRENA, Paul $y$ MARTÍNEZ NAVARRO, Bienvenido, "Paleontología de la Sima de las Palomas de Teba", en WENIGER y RAMOS, Eds., (2014): Sima de las Palomas, Teba (Málaga). Resultados de las investigaciones 2011-2014, Málaga, Ediciones Pinsapar, 2014, 79-91.

FERNÁNDEZ, Santiago, FUENTES, Noemi, CARRIÓN, José, GONZÁLEZ-SAMPÉRIZ, Penélope, MONTOYA, E., GIL-ROMERA, Graciela, VEGA-TOSCANO, Gerardo, RIQUELME CANTAL, José Antonio, "The Holocene and Upper Pleistocene pollen sequence of Carihuela Cave, southern Spain", en Geobios 40, 1, 2007, 75-90.

FERNÁNDEZ RODRÍGUEZ, Luis Efrén, Tipología de las cerámicas lisas de la Cueva de las Palomas (Teba, Málaga), Málaga, Memoria de Licenciatura, Universidad de Málaga, 1986. 
FERRER PALMA, José Enrique y FERNÁNDEZ RUIZ, Juan, "Avance al estudio de la industria en sílex de la Cueva de las Palomas (Teba, Málaga)", en Mainake VIII-IX, 1988, 5-14.

FERRER PALMA, José Enrique y MARQUÉS MERELO, Ignacio, "Avance de las campañas arqueológicas realizadas en la Cueva de las Palomas, Teba (Málaga)", en Baética I, 1978, 195-199.

FINLAYSON, Clive, GILES, Francisco, RODRÍGUEZ VIDAL, Joaquín, FA, Darren, GUTIÉRREZ, José María, SANTIAGO, Antonio, FINLAYSON, Geraldine, ALLUÉ, Ethel, BAENA, Javier, CÁCERES, Isabel, CARRIÓN, José, FERNÁNDEZ-JALVO, Yolanda, GLEED-OWEN, Chris, JIMÉNEZ-ESPEJO, Francisco Javier, LÓPEZ, Pilar, LÓPEZ SÁEZ, José Antonio, RIQUELME, José Antonio, SÁNCHEZ MARCO, Antonio, GILES GUZMÁN, Francisco, BROWN, Kimberly, FUENTES, Noemi, VALARINO, C.A., VILLALPANDO, Antonio, STRINGER, Christopher, MARTíNEZ-RUIZ, Francisca, SAKAMOTO, Tatsuhiko, "Late survival of Neanderthals at the southernmost extreme of Europe", en Nature, 443, 2006, 850-853.

FONTAO REY, María del Mar, La cerámica decorada de la Cueva de Las Palomas (Teba, Málaga), técnicas y estilos. Málaga, Memoria de Licenciatura, Universidad de Málaga, 1986.

GILES PACHECO, Francisco, GUTIÉRREZ LÓPEZ, José María, SANTIAGO PÉREZ, Antonio, MATA, Esperanza, (1998). Avance al estudio sobre poblamiento del Paleolítico superior en la cuenca media y alta del Río Guadalete (Cádiz)", en SANCHIDRIÁN y SIMÓN, Eds., Las Culturas del Pleistoceno Superior en Andalucía, Málaga, Patronato de la Cueva de Nerja, 1998,111-140.

HOFFMANN, Dirk, STANDISH, Chris, GARCÍADÍEZ, Marco, PETTITT, Paul, MILTON, J. Andrew, ZILHÂO, Joao, ALCOLEA, Javier, CANTALEJO DUARTE, Pedro, COLLADO, Hipólito, BALBÍN, Rodrigo, LORBLANCHET, Michel, RAMOS MUÑOZ, José, WENIGER, Gerd-Christian y PIKE, Alistair, "U-Th dating or carbonate crusts reveals Nenadertal origien or
Iberian cave art", en Science 359, 2018, 912915.

HOFFMEISTER, Dirk, ZELLMANN, Stefan, PASTOORS, Andreas, KEHL, Martin, CANTALEJO, Pedro, RAMOS, José, WENIGER, Gerd-Christian, BARTEH, Georg, "The Investigation of the Ardales Cave, Spain -3D Documentation, Topographic Analyses, and Lighting Simulations based on Terrestrial Laser Scanning", en Archaeological Prospection Archaeol, 2015, Doi: 10.1002/arp.1519.

JENNINGS, Richard, GILES PACHECO, Francisco, BARTON, Nick, COLLCUTT, S.N., GALE, R., GLEED-OWEN, Chris, GUTIÉRREZ LÓPEZ, José María, HIGHAM, Tom, PARKER, A., PRICE, C., RHODES, E., SANTIAGO PÉREZ, A., SCHWENNINGER, Jean-Luc, TURNER, E., "New dates and palaeoenvironmental evidence for the Middle to Upper Palaeolithic occupation of Higueral de Valleja Cave, southern Spain", en Quaternary Science Reviews 28, 2009, 830839.

KEHL, Martin, BUROW, Christoph, CANTALEJO DUARTE, Pedro, DOMÍNGUEZ-BELLA, Salvador, DURÁN VALSERO, Juan José, KLASEN, Nicole, MEDIANERO SOTO, Francisco Javier, RAMOS MUÑOZ, José, REICHERTER, Klaus, SCHMIDT, Christoph y WENIGER, GerdChristian, "The Palaeolithic Site Sima de las Palomas de Teba, Southern Spain - Site formation processes and Chronostratigraphy", en BAENA, FERNÁNDEZ y GUERRERO, Eds., VIII Reunión de Cuaternario Ibérico. La Rinconada. EI Cuaternario Ibérico. Investigación en el siglo XXI, La Rinconada, Sevilla, Aequa, 2013, 285289.

KEHL, Martin, BUROW, Christoph, CANTALEJO, Pedro, DOMínGUEZ-BELLA, Salvador, DURÁN, Juan José, HENSELOWSKY, Félix, KLASEN, Nicole, LISTÄDTER, Joerg., MEDIANERO SOTO, Javier, PASTOORS, Andreas, RAMOS MUÑOZ, J., REICHERTER, K., SCHMIDT, Christoph y WENIGER, GerdChristian, "Site formation and chronology of the new Paleolithic site Sima de Las Palomas de Teba, southern Spain, en Quaternary Research 85, Issue 2, 2016, 187-332. 
LEIVA ROJANO, José Antonio, Estudio estratigráfico de la Cueva de las Palomas (Teba, Málaga). Los niveles superiores, Granada, Memoria de Licenciatura, Universidad de Granada, 1977.

MADOZ, Pascual, Diccionario GeográficoEstadístico-Histórico de España y sus posesiones de Ultramar. Málaga, Madrid, 1845-1850. Valladolid, Ámbito Ediciones. 1986.

MEDIANERO SOTO, Francisco Javier, Estado actual en el acondicionamiento, cerramiento y limpieza en la Cueva de las Palomas de Teba (Teba, Málaga), Málaga, Archivo de la Delegación de Cultura de Málaga, Inédito, 2007.

MEDIANERO SOTO, Francisco Javier, "La arqueología en la Escuela Taller Parque Guadalteba. De la formación al empleo y del olvido al desarrollo del patrimonio arqueológico de la Comarca del Guadalteba", en Mainake XXXII, 2010, 1007- 1019.

MEDIANERO SOTO, Francisco Javier, RAMOS MUÑOZ, José, PALMQUIST BARRENA, Paul, WENIGER, Gerd-Christian, RIQUELME CANTAL, José Antonio, ESPEJO HERRERÍAS, María del Mar, CANTALEJO DUARTE, Pedro, ARANDA CRUCES, Antonio, PÉREZ, J.A., FIGUEIRIDO CASTILLO, Borja, ESPIGARES ORTIZ, Patrocinio, ROS-MONTOYA, Sergio, TORREGROSA, Vanessa, LINSTÄDTER, Jorg, CABELLO LIGERO, Lidia, BECERRA MARTÍN, Serafín, LEDESMA CONEJO, Patricia, MEVDEV, Inés., CASTRO, A., ROMERO, M. y MARTÍNEZ NAVARRO, Bienvenido, "The karst site of Las Palomas (Guadalteba Coutry, Málaga, Spain): A preliminary study of its Middle-Late Pleistocene archaeopleontological record", en Quaternary International, 243, 2011, 127-136.

MEDIANERO SOTO, Francisco Javier, RAMOS MUÑOZ, José, CANTALEJO DUARTE, P., DURÁN VALSERO, Juan José, WENIGER, GerdChristian, DOMínGUEZ-BELLA, Salvador y ESPEJO HERRERÍAS, María del Mar, "La ocupación del territorio de la comarca del Guadalteba (Málaga, Sur de España) por sociedades del Pleistoceno", en Menga.
Revista de Prehistoria de Andalucía 3, 2012, 59-82.

MORGADO RODRÍGUEZ, A., "Las primeras sociedades", en GARCIA, MARTÍNEZ y MORGADO, Eds., El bajo Guadalteba (Málaga): Espacio y poblamiento. Una aproximación arqueológica a Teba y su entorno, Málaga, Ayuntamiento de Teba y Diputación provincial de Málaga, 1995, 27-87.

RAMOS MUÑOZ, JOSé, ESPEJO HERRERÍAS, María del Mar, CANTALEJO DUARTE, Pedro, MARTÍN CÓRDOBA, Emilio, MOLINA MUÑOZ, José Antonio, RAMÍREZ TRILLO, Federico, DURÁN VALSERO, Juan José, GRÜN, Rainer, FORD, Derek C., y ALCÁZAR GODOY, José, 1992: Cueva de Ardales (Málaga), su recuperación y estudio, Málaga, Ayuntamiento de Ardales, 1992.

RAMOS MUÑOZ, JOSé, ESPEJO HERRERÍAS, María del Mar, CANTALEJO DUARTE, Pedro, DURÁN VALSERO, Juan José, MARTÍN CÓRDOBA, Emilio y RECIO RUIZ, Ángel, "Cueva de Ardales (Málaga): Geocronología evolutiva y cambios climáticos en el Pleistoceno superior y Holoceno. Los testimonios de su ocupación por formaciones sociales de cazadores, recolectores, tribales y clasistas iniciales", en Mainake XIX-XX, 1988, 17-45.

RAMOS MUÑOZ, José, WENIGER, GerdChristian, CANTALEJO DUARTE, Pedro, ESPEJO HERRERÍAS, María del Mar, Eds., 2014. Cueva de Ardales 2011-2014. Intervenciones arqueológicas, Málaga, Ediciones Pinsapar, 2014.

RAMOS MUÑOZ, José, WENIGER, GerdChristian, PASTOORS, Andreas, LINSTÄDTER, Jörg, BARRENA TOCINO, Antonio, GUTIÉRREZ LÓPEZ, José María, VIJANDE VILA, Eduardo, CANTILLO DUARTE, Juan José, DOMÍNGUEZBELLA, Salvador, MEDIANERO SOTO, Javier, CANTALEJO DUARTE, Pedro, ESPEJO HERRERÍAS, María del Mar, BECERRA MARTÍN, Serafín, CABELLO LIGERO, Lidia, "Tecnología lítica tallada", en WENIGER y RAMOS, Eds, Sima de las Palomas de Teba 2011-2014. Intervenciones arqueológicas, Málaga, Ediciones Pinsapar, 2014, 115-178. 
RAMOS MUÑOZ, José, WENIGER, GerdChristian, Memoria Final de la Actividad Arqueológica de "Excavación y documentación en la Cueva de Ardales 2015, Málaga", original depositado en la Delegación Provincial de Cultura de Málaga, 2016.

RAMOS-MUÑOZ, J., WENIGER. G.-C., CANTALEJO, P., BOLÍN, V., KEHL, M., ESPEJO, M. M., TAFELMAIER, Y., PASTOORS, A., DOMÍNGUEZ-BELLA, S., CABELLO, L., OTTO, T., FERNÁNDEZ-SÁNCHEZ, D., MORENO MÁRQUEZ, A., ROTGÁNGER, M., VIJANDEVILA, E., BECERRA, S., KELLBERT NIELSEN, T., BARRENA-TOCINO, A., ALMISAS-CRUZ, S., CANTILLO-DUARTE, J.J., RIQUELME, J.A., BELTRÁN, A., UZQUIANO, P., RAMOS-GARCÍA, P., BAILÓN, S., ROFES, J. y SÁNCHEZ-MARCO, A. "Excavations in Solutrean levels of Ardales Cave (Málaga, España". En SCHMIDT, I., CASCALHEIRA, J., BICHO, N., WENIGER, G.-C, eds. Human Adaptations to the Last Glacial Maximum. Cambridge Scholar, 2019, 171-187.

RECIO RUIZ, Ángel, "Informe arqueológico del Término Municipal de Teba (Málaga)", en Anuario Arqueológico de Andalucía/1991, III, 1993, 413-418.

RODRIGUEZ VINCEIRO, Francisco y MARQUEZ ROMERO, José Enrique, "Dataciones absolutas para la prehistoria reciente de la provincia de Málaga: una revisión crítica", en Baetica 25, 2003, 313-353.

RUIZ ZAPATA, Blanca y GIL GARCÍA, María José, "Estudio polínico de la secuencia", en
WENIGER y RAMOS, Ed. (2014): Sima de las Palomas, Teba (Málaga). Resultados de las investigaciones 2011-2014, Málaga, Ediciones Pinsapar, 2014, 79-91.

SANCHIDRIÁN, José Luis, "Arte paleolítico de la zona meridional de la Península Ibérica", en Complutum 5, 1998, 163-195.

SANCHIDRIÁN, José Luis, Manual de arte prehistórico, Ariel Prehistoria, 2001.

VALLESPÍ PÉREZ, Enrique, "El Paleolítico inferior y medio en Andalucía", en Homenaje a Luis Siret (1934-1984), 59-66, Sevilla, Junta de Andalucía, 1986, 59-66.

WENIGER, Gerd-Christian y RAMOS MUÑOZ, José, Eds., Sima de las Palomas, Teba (Málaga). Resultados de las investigaciones 2011-2014. Málaga, Ediciones Pinsapar, 2014.

WENIGER, Gerd-Christian, RAMOS MUÑOZ, José, Memoria Final de la Actividad Arqueológica de "Excavación y documentación en la Sima de las Palomas de Teba", original depositado en la Delegación Provincial de Cultura de Málaga, 2016.

WOOD, Rachel, BARROSO RUIZ, Cecilio, CAPARRÓS, Miguel, JORDÁ PARDO, Jesús, GALVÁN SANTOS, Bertila, HIGHAM, Tom, "Radiocarbon dating casts doubt on the late chronology of the Middle to Upper Palaeolithic transition in southern Iberia". Proceedings of the National Academy of Sciences 110, 2013, 2781-2786. 